Instituto Internacional de Investigación y Desarrollo Tecnológico Educativo INDTEC, C.A. DOI: https://doi.org/10.29394/scientific.issn.2542-2987.2017.2.4.5.85-105

OAI-PMH: http://www.indteca.com/ojs/index.php/Revista Scientific/oai

\title{
El Diseño Instruccional como Recurso para el Fortalecimiento de la Identidad Regional
}

\author{
Autores: Carol del Carmen Terán González
} Universidad Nacional Experimental "Rafael María Baralt", UNERMB carolterang@gmail.com; carolteranula@gmail.com Trujillo, Venezuela María Ninoska Suarez Rojas Universidad Politécnica Territorial del Estado Trujillo "Mario Briceño Iragorry", UPTT marianinoskasuarez@hotmail.com Trujillo, Venezuela Sonia María Aguilar Rondón Universidad Politécnica Territorial del Estado Trujillo "Mario Briceño Iragorry", UPTT sonia aguilar1965@hotmail.com

Trujillo, Venezuela

\section{Resumen}

La presente investigación tiene como objetivo fundamental, la elaboración de un diseño instruccional digital que contribuya al fortalecimiento de la identidad regional de los estudiantes. El estudio se apoya en los postulados teóricos de: UNESCO (2004), CBN (1997), Ausubel (1983), Vygotsky, Rodríguez (2001), Careaga (2001), Galvis (1992), Pastorini (2000), Salazar (2008), Rosario (2005), Bernice McCarthy (1987), entre otros. La misma adoptó el tipo de investigación descriptiva, con un diseño de campo, enmarcado en la modalidad de proyecto factible. La población está constituida por 12 docentes, y 40 estudiantes de sexto grado. Para la fase diagnóstica se aplicará un cuestionario con 14 ítems para el personal docente y estudiantes, la validación será de contenido a través del juicio de expertos. Se llegó a la conclusión de la debilidad existente en cuanto a los elementos constitutivos de la memoria histórica como parte de la identidad regional.

Palabras clave: diseño instruccional; material educativo computarizado; identidad nacional.

Fecha de Recepción: 20-10-2016

Fecha de Aceptación: 03-12-2016 


\section{Instructional Design as Resource for the Strengthening of the Regional Identity}

\section{Abstract}

The present investigation takes as a fundamental goal, the elaboration of an instructional digital design that contributes to the strengthening of the regional identity of the students. The study is based on the theoretical postulates of: UNESCO (2004), CBN (1997), Ausubel (1983), Vygotsky, Rodríguez (2001), Careaga (2001), Galvis (1992), Pastorini (2000), Salazar (2008), Rosario (2005), Bernice McCarthy (1987), among others. The same one, adopted the type of descriptive investigation, with a field design placed in the modality of viable project. The population is constituted by 12 teachers, and 40 students of the sixth degree. For the diagnostic phase a questionnaire will be applied with 14 items for teachers and students, the validation will be of content across the experts' judgment. In conclusion, there exists weakness for the constitutive elements of the historical memory as part of the regional identity.

Keywords: design instructional; educational computerized material; national identity. 


\section{Introducción}

El proceso de globalización ha facilitado a los venezolanos el acceso a grandes volúmenes de información, sobre todo con el advenimiento de las tecnologías y su penetración en múltiples aspectos de la vida cotidiana, como la salud, el comercio, las finanzas, el hogar, la escuela y la cultura, situación está que ha requerido de formación y transformación de las instituciones para lograr que el país no se quede inerte ante los avances mundiales.

Fernández y otros (2005: 25) señalan que "el inicio de un nuevo siglo, caracterizado por la era de la información y globalización, favorece el intercambio cultural, pero también se invaden espacios vertiginosamente", por lo que debemos estar atentos y preparados para saber manejarlo y más aún aprovecharlo, en este sentido Montiel (2012: 19), plantea que

la arremetida de la industria cultural globalizadora impone líderes modélicos reñidos con nuestra tradición y promueve géneros artísticos exógenos, que no nos representan; los medios de comunicación y las TIC pueden ser el vehículo importante que promueva el reforzamiento de valores culturales [...] sin identidad regional no hay sentido de pertenencia. No hay sentido de arraigo a la nación venezolana.

Situaciones como estas vienen amenazando desde hace años, pues ya para 1951 Don Mario Briceño Iragorry en su obra Mensaje sin Destino atribuía a la falta de sentido histórico de los pueblos gran parte de la crisis del país, por no estar preparados para entender "la historia como sentido de continuidad y de permanencia creadora" Monte Ávila editores (1980, pág. 46), este planteamiento lo hace reconociendo en su tiempo al igual que lo hacemos hoy día la importancia del avance en términos de industria y comercio y de saber de la existencia de otras culturas.

No obstante, trata especialmente la necesidad de buscar de esos valores legítimos, que hoy entendemos como identidad nacional y que están 
referidos al conjunto de elementos antropológicos y culturales que caracterizan y hacen diferentes a los ciudadanos de un país y que pueden ayudar a satisfacer los deseos naturales de desarrollo de los pueblos

Para Gruzinski (2000, pág. 52) citado por Monsalve (2012). La identidad es lo que "asigna a cada ser o a cada grupo humano unas características y unas aspiraciones que también están determinadas y que, supuestamente, se basan en un sustrato cultural estable", la materialización de esta conceptualización es lo que ha venido ocupando la labor de muchos hombres y gobiernos y que hoy la encontramos dentro de la legislación de nuestro país, entre los derechos culturales y educativos que el estado está obligado a garantizar y la escuela a promover; para Ibídem (2012:22) la identidad es "el sentimiento que se va construyendo desde la escuela, de valoración y afecto a nuestro entorno social, es el que nos define y diferencia categóricamente de otros pueblos".

Concientes de esta situación de ayer y hoy, es necesario activar estrategias que puedan coadyuvar al control de la misma y al fortalecimiento de nuestros valores y cultura, potenciando el sentimiento nacionalista para recibir con madurez identitaria y amor patrio manifestaciones culturales de otras latitudes, destacando que la escuela juega un papel determinante en esta labor.

Ibídem (2012: 23) plantea que "para lograr generar una identidad regional, estamos llamados a hacerlo desde la escuela y desde los medios de comunicación masivos, en especial desde las TIC, que forman parte del quehacer cotidiano de los ciudadanos de todos los grupos etéreos"

Entendiendo la identidad regional como el "conjunto de símbolos, formas artísticas, costumbres, imaginario colectivo y tradiciones que otorgan distinción a los ciudadanos de cada región", es determinante formar una sólida 
identidad regional que siente las bases para una gran identidad nacional, no olvidemos que fortaleciendo las partes se garantiza la victoria del todo.

La escuela tiene especial responsabilidad en la creación del sentido de pertenencia y de amor a la patria, por ser un espacio determinante en la formación de conceptos y valores sociales, tal como lo establece el artículo 102 de la CRBV, esta puede llegar a ser salvadora o verdugo de la identidad de los pueblos y del valor de su gente, por lo que se requiere revisar que se está haciendo en el interior de éstas por el desarrollo de la conciencia patriótica y republicana consustanciada con la identidad local, regional y nacional, tal como lo establecen los fines y principios del sistema educativo bolivariano (SEB), entre los cuales también se propone el empleo de las Tecnologías de la Información y la Comunicación (TIC), desde un enfoque social, como herramienta de trabajo y recurso para el aprendizaje.

Siendo los niños, niñas y adolescentes las poblaciones más vulnerables a la influencia de manifestaciones culturales ajenas a su entorno (extranjeras), por considerarlas de actualidad, porque así las presentan los medios de comunicación masiva, es necesario generar estrategias que canalicen la situación, tal como está sucediendo en los primeros 3 grados de la educación básica con la implementación del proyecto Canaima Educativo.

En este sentido precisamente apunta esta investigación, la cual parte del demérito de lo regional, como problemática reflejo de que los estudiantes no se identifican a plenitud con su estado y localidad porque desconocen mucho de las riquezas, bondades y acervo cultural de estas, situación que incide directamente en la conformación de valores sociales, a saber, la identidad regional del merideño.

Conviene entonces trazar una interrogante en relación a lo señalado hasta aquí: ¿La incorporación de diseños instruccionales digitales sirve de puente para el aprendizaje significativo de elementos determinantes en la identidad regional y nacional?. 


\subsection{Propósito general}

Elaborar un diseño instruccional digital prototipo como recurso para el fortalecimiento de la identidad regional de los estudiantes de educación básica.

\section{Bases Teóricas}

Los postulados teóricos y enfoques considerados para soportar esta investigación se encuentran en consonancia con los adoptados por el Ministerio del Poder Popular para la Educación en el diseño curricular para el sistema educativo bolivariano (SEB) y todos aquellos aportes de paradigmas psicológicos aplicados a la educación. Entre los fines de la Educación Primaria Bolivariana (2007: 18) está formar niños y niñas

"con una conciencia que les permita comprender, confrontar y verificar su realidad por sí mismas; que aprendan desde el entorno, para que sean cada vez más participativos, protagónicos y corresponsables de su actuación en la escuela, la familia y la comunidad"

Se aspira formar una actitud humanista, crítica y reflexiva en los y los estudiantes, consustanciada con la identidad local, regional y nacional, donde éstos sean participantes activos en la construcción de sus conocimientos.

\subsection{Enfoque constructivista de la educación}

Para Díaz (2004) el constructivismo:

Es una teoría que equipara el aprendizaje con la creación de significados a partir de experiencias; la cual no niega la experiencia del mundo real, pero sostiene que lo conocido de él nace de la propia interpretación de nuestras experiencias los seres humanos crean conocimiento (pág. 53).

Este enfoque sostiene que se construyen interpretaciones personales del mundo apoyadas en las experiencias e interacciones particulares, concepciones como esta son las que actualmente se adoptan en la educación 
por estar cónsonas con los fines de la educación bolivariana y con el tipo de aprendizaje que se debe promover para lograr la formación integrar del estudiante y esa actitud crítica y responsable socialmente que nuestras leyes (CRBV y LOE) aspiran y el país necesita.

El objetivo de esta investigación se sostiene también en esta teoría, considerando que el MEC diseñado consiste en un recorrido virtual por el municipio al que pertenece el estudiante y dentro del cual encontrará muchos elementos que ya están registrados entre sus experiencias y que le harán evocar conocimientos y construir otros nuevos, a partir de los existentes y de la nueva información incorporada a sus estructuras mentales.

\subsection{Aprendizaje Significativo}

Teóricos como Ausubel (1983.157), enfatizan en que el aprendizaje implica una "reestructuración activa de las percepciones, ideas, contenidos y esquemas que el aprendiz posee en su estructura cognitiva", asimismo éste concibe al estudiante como un "procesador activo de la información" y sostiene que el aprendizaje es sistemático y organizado, pues es un fenómeno complejo que no se reduce a simples asociaciones memorísticas.

Osorio, R. (2007: 65) David Ausubel propuso el término «Aprendizaje significativo» para designar el proceso a través del cual la información nueva se relaciona con un aspecto relevante de la estructura del conocimiento del individuo. A la estructura de conocimiento previo que recibe los nuevos conocimientos «concepto integrador». El aprendizaje significativo se produce por medio de un proceso llamado Asimilación. En este proceso, tanto la estructura que recibe el nuevo conocimiento, como este nuevo conocimiento en sí, resultan alterados, dando origen a una nueva estructura de conocimiento.

La teoría propuesta por Ausubel ha tomado gran valor para la educación venezolana, siendo adoptada como sustento teórico dentro del Currículo 
Básico Nacional, por considerarla como una propuesta que junto con otras teorías (Psicogenética y socio cultural), contribuirían a la formación integral de nuestros estudiantes, esta teoría se caracteriza por dar valor a las diferencias individuales del estudiante, atendiendo a sus experiencias y haciéndolo participante activo del proceso educativo, por considerarlo en plena capacidad de construir su propio conocimiento.

Los materiales educativos computarizados responden a esta premisa de hacer del estudiante un participante activo en los procesos educativos, los MEC son utilizados directamente por el estudiante, éste puede hacer el recorrido de manera individual, guiado en unos casos por el docente, pero siguiendo su propio ritmo, las diferencias individuales son especialmente atendidas por los MEC multimedios, pues responden a la variedad de formas de aprender al mostrar la información de diferentes formas, imágenes para los visuales, sonidos para los auditivos y de manera virtual pudieran atender también a los más kinestésicos.

Atendiendo a los principios propuestos por esta teoría y la relación que establece entre aprendizaje y desarrollo, se pueden caracterizar sus fortalezas de la siguiente manera:

- El aprendizaje es personal, ya que depende de los recursos cognitivos del alumno, de sus experiencias, necesidades y expectativas.

- La incorporación de nueva información a la estructura cognitiva del estudiante modifica la existente y lleva a la construcción de una nueva, a la reconstrucción de esquemas de conocimiento, es decir, se establece una relación entre los nuevos conocimientos y los ya adquiridos, lo que garantiza una retención más duradera de la información. 
En este sentido los llamados conocimientos previos y la experiencia de quien aprende, resultan determinantes en la construcción de nuevos conocimientos.

\subsection{Enfoque Socio-Cultural: Lev S. Vygotsky.}

Entre las orientaciones que sustentan el SEB el trabajo cooperativo y colaborativo cobra especial importancia, se habla del vivir y convivir como unas de las capacidades del nuevo republicano, concibiendo la educación como un "proceso social que emerge de la raíz de cada pueblo, orientado a desarrollar el potencial creativo de cada ser humano" (pág. 42), planteando la posibilidad de "aprender con los demás" (pág. 51), colocando al docente como "activadores, activadoras, mediadores y mediadoras de los saberes, el sentir y el hacer social y cultural, y el procesos de apropiación de los aprendizajes por parte de los y las estudiantes" (pág. 50); concibiendo a la escuela como centro del quehacer comunitario y como espacio de comunicación (entre otros), sumando relevancia al contexto y a las relaciones de intercambio.

A partir de la tesis presentada por Vygotsky, Rodríguez (2001: 52), plantea: La formulación de la ley es clara con respecto a que el desarrollo cognoscitivo, emerge de la interacción social y de la mediación semiótica que caracteriza la vida en la sociedad. En el curso de las interacciones con las otras personas, el individuo entra en contacto y aprende a utilizar las herramientas (instrumentos y artefactos) y los sistemas simbólicos de su cultura (entre los que se encuentra el lenguaje) el aprendizaje estimula y activa una variedad de procesos mentales que afloran en el marco de la interacción con otras personas... 
Atendiendo a los aportes de la teoría de Lev Vygotsky a la educación y
la relación que establece entre aprendizaje y la interacción con otros, se puede caracterizar:

- La Interacción y cooperación social es importante en la construcción de los aprendizajes y en desarrollo del estudiante.

- El hombre busca su evolución y bienestar, por lo que actúa sobre la realidad para adaptarse, para transformarla y transformarse a sí mismo.

- Da especial Importancia al contexto, ya que a partir del contacto con este se potencia su desarrollo y aprendizaje.

- Plantea la formación social de la mente, el convivir y el hacer, determinan el conocer.

- El estudiante puede lograr más con la ayuda de otros (nivel de desarrollo potencial) que por sí solo (nivel de desarrollo real).

- El estudiante construye a partir de su realidad; el entorno del estudiante influye en su personalidad y devenir, más aún, lo define.

La visión constructivista del aprendizaje según Vygotsky sostiene que la finalidad de la educación es promover los procesos de crecimiento personal del estudiante en el marco de la cultura del grupo al que pertenece. En consecuencia, los procesos de formación deben promover tanto la socialización como la individualización que permita a los estudiantes construir una identidad personal en el marco de un contexto social y cultural determinado.

\subsection{Teoría Cibernética}

Según Couffignal en Careaga (2001: 36), el campo de la pedagogía desde la perspectiva de la cibernética reúne "Los mecanismos mediante los 
cuales un educando adquiere conocimientos y el análisis crítico de las finalidades por las que ese mecanismo se pone en funcionamiento".

En este sentido la presencia del docente como guía del proceso, la pertinencia y contextualización del material educativo, la consideración de las características y necesidades de los estudiantes, los principios establecidos en el CBN y el modelo propuesto por Galvis (1992) para el diseño de software educativo, garantizará la disposición de las oportunidades que en términos de construcción del aprendizaje promoverá el material, especialmente por la consideración y el respeto a las diferencias individuales.

Couffignal en Careaga (2001: 53) "Dentro del funcionamiento del mecanismo pedagógico, el ritmo de presentación de las informaciones sucesivas de una lección debe ser establecido en concordancia con el tiempo de respuesta del alumno". Las características de los estudiantes de una sección son diversas, algunos experimentan mejores resultados a través del texto, otros son más auditivos, otros visuales y muchos kinestésicos, en la educación tradicional ha sido difícil para el docente atender todas estas características, por lo que difícilmente todos los estudiantes logran asimilar la totalidad de la información.

\subsection{La integración de las Tecnologías de Información y Comunicación (TIC) en la educación.}

Hoy las instituciones educativas no son entes aislados, sino que pueden estar en permanente conexión con otras fuentes de información a través del computador como medio para acceder a la información y a la comunicación. Las redes de comunicación se irán expandiendo cada vez más e igualmente la elaboración de programas multimedios y, aunque la producción de programas de realidad virtual en todavía incipiente en el país y los equipos costosos, la situación está cambiando gracias al desarrollo vertiginoso de las posibilidades que ofrece el gobierno nacional a través del MPPE, como son 
los casos de la instalación de los Centros Regionales de Desarrollo de Contenidos Educativos Digitalizados y la distribución de computadores portátiles Canaima para la educación primaria.

Conviene destacar que para la integración de las Tecnología de la Información y Comunicación (TIC), el docente no tiene que adquirir necesariamente una formación como expertos informático o programador, sino como usuario, promotor de su uso y diseñador instruccional, que a través de los proyectos de aprendizaje integre las TIC al proceso educativo.

Semenov (2006: 185) plantea al respecto que las TIC no son una materia cerrada o autónoma que puede enseñarse y prenderse en forma independiente de las demás materias. Por el contrario, las TIC, por su propia naturaleza, son una materia que debería tratarse como interdisciplinaria, integradora y transcurricular.

En este entendido se asume que los computadores además de ser un objeto de estudio en sí mismos, constituyen un medio para la enseñanza y el aprendizaje, logrando así el desplazamiento del uso instrumental de la tecnología hacia la utilización pedagógica dentro del proceso de desarrollo de las instituciones educativas, tal como lo plantea el MPPE en los objetos del proyecto Canaima educativo (2010) "Transformar la praxis docente con el uso humanista, crítico y creativo de las tecnologías de la información libres" (pág. 7). Sin embargo, es difícil determinar todos los efectos que las tecnologías informáticas pueden tener sobre la educación porque, entre otras razones, no se ha desarrollado suficiente investigación para documentar estos efectos.

\subsection{El Diseño Instruccional}

La complejidad de la producción de Materiales Educativos Computarizados (MEC) estriba en la responsabilidad no sólo de llevar las tecnologías al aula, sino en la necesidad de tomar decisiones en torno a los contenidos y su presentación, las estrategias de enseñanza y de aprendizaje, 
tal como se realiza para la incorporación de recursos para el aprendizaje de tipo tradicional, teniendo siempre presente que su objeto es facilitar el proceso de asimilación y organización de la información en las estructuras mentales de los estudiantes. En este sentido el diseño instruccional es un proceso que viene siendo formalmente aplicado desde los años 60 , y que hace referencia a la especificación y disposición de todos los elementos que participan en el proceso educativo, en función del logro de aprendizajes y la orientación de la acción docente.

Para Dorrego y García (1991:123)

"constituye el proceso mediante el cual se analizan las necesidades y metas de la enseñanza, a partir de ese análisis se seleccionan y desarrollan las actividades y recursos para alcanzar esas metas, y así los procedimientos para evaluar el aprendizaje de los alumnos y revisar la instrucción"

Para el Diseño Instruccional además de contemplar todos los elementos que intervienen en el proceso educativo y organizarlos a manera de pautas, se debe partir de principios psicoeducativos y del análisis o diagnóstico de la situación de entrada y de la audiencia, asimismo todo esto debe estar enmarcado en un modelo o metodología didáctica. En este aparte conviene destacar la existencia de varios modelos de Diseño Instruccional, tales como: 4 MAT Bernice McCarthy, Dick y Carey, Jerrold y Kemp, Modelo inductivo, Modelo de adquisición de conceptos, Modelo integrativo, Modelo de enseñanza directa, Modelo de exposición y discusión, Modelo de indagación, Modelo de aprendizaje cooperativo.

De esta revisión y considerando la audiencia, el contenido y el resto de los elementos hasta ahora mencionados y que son medulares para el logro de aprendizajes significativos, se opta por el modelo propuesto por Bernice McCarthy, llamado 4 MAT, el cual se encuentra en consonancia con lo establecido en el diseño curricular del sistema educativo bolivariano. 


\section{Marco Metodológico}

\section{Tipo de Investigación}

Para llevar a cabo el proceso de investigación es necesario tener claros, tanto los propósitos de la investigación, como el conocimiento que se desea obtener de la misma, a fin de determinar el tipo de investigación que se va a realizar. En base a lo señalado en el Manual de Trabajos de Grado de Especialización y Maestría y tesis de Doctorado, publicado por la UPEL (2012), se ubica en el tipo de investigación de los Proyectos Factibles, a tendiendo a que se refiere a la formulación de una propuesta de un diseño instruccional, para el desarrollo de un Material Educativo Computarizado, con apoyo documental y de campo, que tiene como propósito contribuir a cubrir los requerimientos de un grupo social específico.

El Proyecto Factible consiste en la investigación, elaboración y desarrollo de una propuesta de un modelo operativo viable para solucionar problemas, requerimientos o necesidades de organizaciones o grupos sociales; puede referirse a la formulación de políticas, programas, tecnologías, métodos o procesos. El proyecto debe tener apoyo en una investigación de tipo documental, de campo o un diseño que incluya ambas modalidades. (pág. 36)

Para la realización de esta investigación se parte del diagnóstico tomado in situ, en el campo mismo donde luego será aplicado el material, según la propuesta, para el diseño instruccional se utiliza el modelo propuesto por McCarthy y para el diseño del MEC la metodología de Galvis. Esta investigación hace uso del diseño de campo, por cuanto éste diseño brinda la oportunidad de recoger la información directamente del ambiente en estudio, el respecto señala Balestrini (1998) "permite [...] observar y recolectar los datos directamente de la realidad, en su situación natural”. (pág. 119). 


\section{Instrumentos y técnicas para la recolección de la información}

Análisis documental de las fuentes bibliográficas: Este permite abordar y desarrollar la parte o sustento teórico de la investigación, ésta se lleva a cabo a través de la observación documental, el resumen analítico.

\section{Técnica de entrevista}

La entrevista o dialogo intencional, se realiza con el propósito de obtener información relevante para la investigación en función de sus objetivos, en este caso, cumple una función diagnóstica y orientadora, éstas son informales, a partir de preguntas abiertas y del diálogo directo y espontáneo que lleva a la interacción personal.

\section{Conclusiones}

Se puede afirmar a partir de la investigación que efectivamente existe un desconocimiento por la historia y otros elementos del entorno, aunque los estudiantes dan valor a lo que conocen y de lo que participan, como es el caso de las fiestas religiosas y del cultivo de la tierra; en cuanto al uso de las tecnologías todos coinciden en la necesidad de su incorporación a los procesos de enseñanza y aprendizaje como un recurso altamente motivador, asimismo hablan de su deseo de conocer más sobre el municipio a través de un material multimedios, de manera virtual.

En este sentido la investigación se basó en la propuesta de incorporación de un material educativo computarizado de tipo simulador y con ejercicios prácticos que muestre las bondades y riquezas del municipio Rangel y sus poblados, específicamente elementos como historia, geografía, economía, gastronomía, festividades, gentilicio y leyendas, todos esos elementos que demuestran las grandezas y riquezas de una tierra, que hacen que su gentilicio la conozca y sienta orgullo, porque sólo lo que se conoce y se valora por grande, se quiere, respeta y resguarda. 
La promoción y trabajo de contenidos como los de ciencias sociales sujetos históricamente la revisión y lectura de largos textos y tratamientos tradicionales a través de recursos multimedios hace más agradable su tratamiento, considerando que la virtualidad lleva al aula la realidad del entorno pensada y organizada desde la formalidad de lo académico curricular, atendiendo además las características de cada estudiando, a sabiendas de que unos son más visuales y otros más auditivos, asimismo permite avanzar y aprender a ritmo propio y contando siempre con la orientación del docentes y hasta de los compañeros más aventajados, en pro de un trabajo colaborativo y cooperativo para la construcción de sus conocimientos.

Durante la intervención se logra la promoción de opiniones más favorables hacia ciertos contenidos, lo cual contribuyo a su vez a la disposición de los estudiantes hacia la construcción de nuevos conocimientos, mediante el uso de las tecnologías, la indagación, la toma de decisiones, el trabajo colaborativo y cooperativa, la investigación y la producción.

Considerando lo anterior se hace necesario que el proceso de enseñanza y aprendizaje sea reorientado en cuanto a las estrategias metodológicas y el uso de determinados recursos, promoviendo el uso de las tecnologías y el trabajo protagónico de los estudiantes, donde el eje central del proceso sea este y su principal objetivo investigar y construí un aprendizaje más significativo, y esto sólo será posible a partir de la consideración de sus capacidades intelectuales, sus inquietudes y necesidades, sólo así lograremos despertar en ellos el deseo de conocer, aprender, aportar y cuidar de su entorno, por ser suyo y por sentirse a su vez parte importante de él.

En este caso el desarrollo del diseño instruccional para el material educativo "Viaje virtual por el municipio Rangel", concebido para el fortalecimiento de la Identidad regional de los estudiantes de sexto grado, se presenta como una herramienta útil para despertar en los estudiantes el deseo por conocer más sobre su legado histórico, sus raíces y todo aquello que 
contribuye y ha hecho posible su devenir y presente, logrando contribuir además a la consolidación de una verdadera conciencia local, regional y nacional.

El Diseño instruccional del material educativo computarizado, se fundamenta la activación de todos los procesos cognoscitivos a favor de aprendizajes más significativos que parten de la construcción del propio conocimiento y el aprovechamiento del trabajo colaborativo y cooperativo o de esa zona de desarrollo más próxima, asimismo en criterios e indicadores que permiten evaluar las dimensiones pedagógicas del aprender para la formación integral del Ser, que funciona como parámetro para determinar los progresos y dificultades de los estudiantes, estableciendo valoraciones de su progreso en la asimilación de los aprendizajes y en función de su desempeño.

\section{Referencias}

Ausubel y Otros (1983). Psicología educativa, Segunda Edición. México: Editorial Trillas.

Balestrini, M. (2009). Como de elabora un proyecto de investigación. Séptima edición. República Bolivariana de Venezuela. Servicio editorial Consultores asociados.

Díaz y Otros. (2002). Estrategias docentes para un aprendizaje significativo. McGraw-Hill, México.

Dorrego E. y García (1991). Dos Modelos para la Producción y Evaluación de Materiales Instruccionales. Edic. Facultad de Humanidades y Educación. Caracas.

Fernández, O. Lúquez, P. Paredes, I. Sansevero, I. (2005). Inserción legal y curricular de la dimensión identidad nacional en la educación básica, Encuentro Educacional, Universidad del Zulia. Escuela de Educación. Facultad de Humanidades y Educación. Estado Zulia Venezuela. 
Galvis, A. (1992). Ingeniería del Software Educativo. Bogotá. Semenov, A. (2006). Las tecnologías de la información y la comunicación en la enseñanza, Manual para docentes o Cómo crear nuevos entornos de aprendizaje abierto por medio de las TIC. Ediciones TRILCE. 


\section{Carol del Carmen Terán González \\ e-mail: carolterang@gmail.com; carolteranula@gmail.com}

Nacida en la ciudad de Valera, Edo Trujillo, Venezuela.

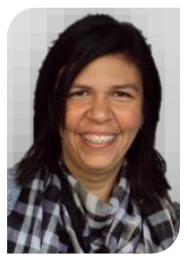

Cursó estudios de Doctorado en Educación en la Universidad Rafael María Baralt, posee Maestría en Docencia para la Educación Superior en la misma universidad, Maestría en Literatura Latinoamericana en la Universidad de los Andes y Licenciatura en Educación Mención Castellano y Literatura ULA-NURR investigadora del Centro de investigaciones literarias y lingüísticas Mario Briceño Iragorry. (CILL) ULANURR, Jefe de la unidad de investigación y proyecto de la Casa de Historia Trujillo, articulista e investigadora con 14 años de servicio en el área de educación y literatura. Ponente en eventos nacionales e internacionales. Docente invitada en pregrado y Postgrado, ULA, UNERMB, UNESR. Coordinadora de la Línea de investigación Memoria, Educación y Discursos emergentes (UNERMB), miembro de la Línea de investigaciones Ciudadanía, hermenéutica y proyectos sociales. (UNERS). Ha realizado artículos en distintas revistas arbitradas del país entre ella Sapiencia, Cifra Nueva, Revista de Cultura Centro Nacional de Historia, Revista de Ingeniería UVM, entre otras. 


\section{María Ninoska Suarez Rojas \\ e-mail: marianinoskasuarez@hotmail.com}

Nacida en la ciudad de Flor de Patria, Edo Trujillo,

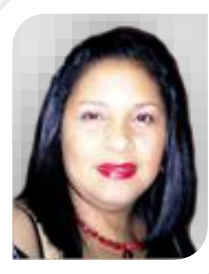

Venezuela. Doctorante en Educación Universidad

Rafael María Baralt, Maestría en Gerencia Empresarial, (URBE). Licenciado en Administración ULA - NUERR.

Docente Contratado Adscrita al Departamento de Administración /Jefe Dpto. de Formación del Docente Universitario. Universidad Politécnica Territorial del Estado Trujillo "Mario Briceño Iragorry" Investigadora y tutora de trabajos de pregrado y Post Grado. Miembro de la Línea de investigación Memoria, Educación y Discursos emergentes (UNERMB). 


\section{Sonia María Aguilar Rondón \\ e-mail: sonia aguilar1965@hotmail.com}

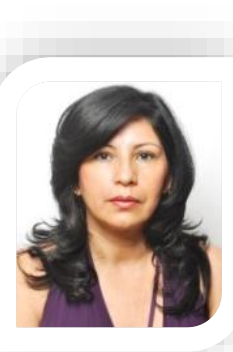

Nacida en la ciudad de Valera, Edo Trujillo, Venezuela.

Doctorante en Educación Universidad Rafael María

Baralt, Maestría en Docencia para la Educación

Superior en la misma universidad, Licenciado en

Educación Mención Castellano y Literatura ULA-NURR. Licenciada en Administración de Empresa ULA-NURR.

Magister en Administración Universidad Rafael María Baralt. Docente de Media General del Ministerio de Educación con 17 años de servicio. Docente Instituto Universitario de Tecnología del Estado Trujillo "Don Rómulo Betancourt" Investigadora y tutora de trabajos de pregrado y Post Grado. Miembro de la Línea de investigación Memoria, Educación y Discursos emergentes (UNERMB).

El contenido de este manuscrito se difunde bajo una Licencia de Creative Commons ReconocimientoNoComercial-Compartirlgual 4.0 Internacional 